\title{
What type of mattress should be chosen to avoid back pain and improve sleep quality? Review of the literature
}

\author{
Gianfilippo Caggiari ${ }^{*}$ (D), Giuseppe Rocco Talesa ${ }^{2}$, Giuseppe Toro ${ }^{3}$, Eugenio Jannelli ${ }^{4}$, Gaetano Monteleone ${ }^{5}$ and \\ Leonardo Puddu ${ }^{6}$
}

\begin{abstract}
Energy spent during daily activities is recuperated by humans through sleep, ensuring optimal performance on the following day. Sleep disturbances are common: a meta-analysis on sleep quality showed that 15-30\% of adults report sleep disorders, such as sleep onset latency $(\mathrm{SOL})$, insufficient duration of sleep and frequently waking up at night. Low back pain (LBP) has been identified as one of the main causes of poor sleep quality. Literature findings are discordant on the type of mattress that might prevent onset of back pain, resulting in an improved quality of sleep. We conducted a systematic literature review of articles published until 2019, investigating the association of different mattresses with sleep quality and low back pain. Based on examined studies, mattresses were classified according to the European Committee for Standardization (2000) as: soft, medium-firm, extra-firm or mattresses customized for patients affected by supine decubitus. A total of 39 qualified articles have been included in the current systematic review. Results of this systematic review show that a medium-firm mattress promotes comfort, sleep quality and rachis alignment.
\end{abstract}

Keywords: Mattress, Back pain, Sleep quality, Sleep, Quality of life

\section{Introduction}

Human beings usually spend around a third of their lifetime sleeping [1], even though this enables individuals to satisfactorily exploit the remaining two-thirds of the day only if sleep was free from disturbances or interruptions. In the past years, an increasing number of people have been complaining of sleep disorders [2].

Although optimal sleep duration is between 7 and $8 \mathrm{~h}$ per day, it has been highlighted that a reduction in sleeping hours has occurred, due to work habits or overall changes in lifestyle. A reduction in sleep hours or sleep quality inevitably has negative impacts on individuals' health, as well as on life and mood quality [3].

\footnotetext{
*Correspondence: gianfilippocaggiari@gmail.com

${ }^{1}$ Orthopaedic and Traumatology Department, Università degli Studi di

Sassari, 07100 Sassari, Italy

Full list of author information is available at the end of the article
}

Low back pain should be taken into consideration among factors that reduce sleep quality.

Although several studies acknowledge the significance of mattresses for sleep quality, there is no common agreement on the optimal design of a mattress to alleviate or prevent cervical or low back pain. Detrimental effects on health led us to analyse which mattress might act as a solution to these problems [4].

Mattress firmness seems to play a leading role as different studies show that medium-firm surfaces might effectively reduce pain in individuals complaining of back pain [5].

An increasing number of companies are promoting their mattresses claiming that they might be able to improve sleep quality and quality of life as a consequence, pretending that their mattresses are "orthopaedic mattresses" with therapeutic properties. However, such claims are not supported by enough evidence. 
Several studies in the literature aimed to assess which could be the best mattress to prevent back pain and improve sleep and life quality; however, the lack of univocal findings suggests that additional research on this issue is warranted. Therefore, the aim of this review is to evaluate available studies so as to understand which mattresses can effectively reduce back pain or prevent its onset. In this way, healthcare professionals will be able to recommend to patients the correct type of mattress to limit or avoid back pain symptoms, thus providing them with benefits in terms of life quality.

\section{Materials and methods}

Studies carried out between 2000 and 2019 have been included in the current review. Mattresses analysed in the studies examined by our research group have been classified according to the European Committee for Standardization (2000) as soft, medium-firm, extra-firm or customized to avoid supine decubitus.

The databases searched include the Cochrane Library, PubMed (Digital Biomedical Archives and Health Sciences of the US National Institutes of Health), Google Scholar, Web of Science and Scopus.

The selection of search terms was tailored to match the search tools of each database, using MeSH terms to search in the databases Science Direct, PsycINFO, EMBASE, PubMed, Google Scholar, Web of Science, Scopus and Cochrane Collaboration. The following terms were used as descriptors: mattress and ergonomics, mattress and pain, mattress and vertebral column, mattress and sleep, mattress and quality of life.

Articles were sourced by searching the databases using the search strategy, or by searching for "similar articles". Inclusion criteria for the articles were publication in English, being published between the years 2000 and 2019 and studies conducted on adults over 18 years of age.

The first screening of articles was carried out by reading the titles and abstracts; those that had no relation to the subject, considering the inclusion and exclusion criteria, were excluded. A total of 323 potentially relevant papers were identified in the five investigated databases; these articles were selected by eliminating those with titles that did not correspond with the search, for example concerning certain categories (athletes, children, hospitalized adults), or were aimed at the study of certain pathologies (sudden death, ulcers, asthma, and other pathologies not related to our study), excluding 194 duplicates. After abstract analysis and reading, 70 articles were selected for full reading, after which 31 articles were excluded. Articles were excluded if they did not account for any biomechanical measurements or investigations; for example, some excluded articles only dealt with posture behaviour, cardiovascular and pulmonary measurements, questionnaires, temperature and humidity. Articles on medical-use mattresses, particularly those describing anti-pressure sore mattresses, were also excluded.

There was a total of 39 articles for analysis. The process of selecting studies is shown in Fig. 1, using a Preferred Reporting Items for Systematic Reviews and Meta-Analyses (PRISMA) flowchart.

A data extraction table was designed to collate information from the studies. For the analysis of the studies, an instrument composed of the following items was used for obtain data: author name, study type, research designs, levels of evidence (Melnyk \& Fineout-Overholt, 2005), populations, samples, results and conclusions. These research characteristics are presented in Table 1.

Level of evidence: 1 article was classified as evidence I (systematic review with and without meta-analysis); $7^{*}$ as evidence II (randomized controlled clinical trial); $27^{*}$ as evidence III (controlled clinical trial without randomization) and $4^{*}$ as evidence level VI (evidence from a single descriptive or qualitative study). Each article has been carefully analysed by two independent reviewers to determine whether it was compliant in terms of inclusion and to evaluate methodological quality. In case of discrepancy between reviewers' evaluations, a third reviewer intervened to analyse the controversy.

\section{Results}

A total of 39 articles were considered compliant with inclusion criteria. Articles included in the current review and main results are presented in Table 1.

Such articles underlined the association between back pain decrease and mattress characteristics, in terms of design and firmness, that promote sleep quality and correct column alignment. Evaluating articles on effective pain relief deriving from use of specific mattresses, three crucial results emerged: three types of mattresses showed the ability to achieve more effective pain relief in study participants.

Some authors declared that a mattress with intermediate firmness might reduce back pain [6-10]. In the literature, some authors recommended mattresses with an air overlay system to reduce pain, while others showed that variation of temperature can promote sleep [11-14].

Jacobson et al. conducted a study comparing back pain, shoulder pain, spinal stiffness, quality, comfort and sleep efficiency in volunteers who had been usually sleeping on commercial spring mattresses (phase 1) and rested for 28 days on medium-firm mattresses (phase 2). Jacobson et al. used a pre-test and a post-test after the experimental phase, using symptomatic patients as a control. In all cases, independently from initial sleep control, benefits were observed as a result of using a medium-firm 


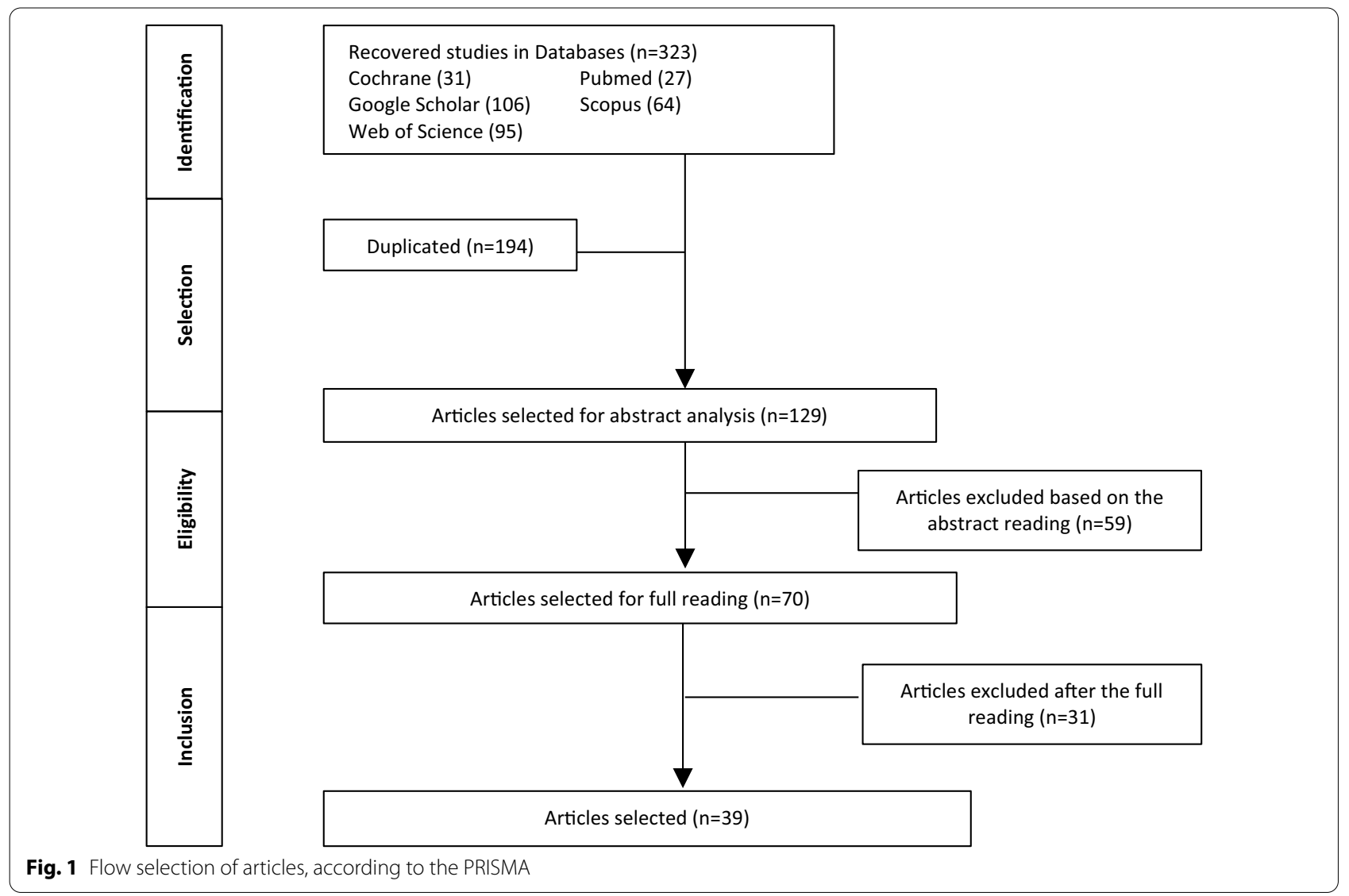

mattress, independently from age, weight, height, and body mass index (BMI). Besides, improvement appeared to be progressively increasing between the first and the fourth week since adoption of new mattresses [5].

Kovacs et al. conducted a double-blind multicentric controlled study evaluating 313 adults diagnosed with non-specific chronic low back pain upon waking up. Results showed that, even though improvement was observed using both mattresses, patients with mediumfirm mattress reported a higher level of improvement both in terms of pain and disability. Thus medium-firm mattresses are recommended to patients suffering from non-specific chronic low back pain.

Some authors studied the efficacy of overlay systems made by using different techniques and materials. Specifically, Monsein et al. carried out a study on 30 patients diagnosed with severe low back pain who did not suffer from sleep disorders or sleep apnea syndrome.

Some authors studied the efficacy of overlay systems made by using different techniques and materials. Final results showed a significant benefit in terms of pain symptoms and sleep quality in patients who had slept on this kind of mattresses [11]. Similar results for what concerns pain decrease and sleep quality were reached by using an air-filled mattress low-pressure fixed overlay (Repose). A study from Price et al. was based on this type of overlay used for 4 weeks by 19 patients affected by chronic low back pain and sleep disorders. Results were statistically significant in terms of decrease in night awakenings as well as for sleep quality and relieved low back pain [12].

Body temperature is among parameters taken into consideration. Raymann group evaluated sleep quality in response to manipulating external body temperature by using a thermal suit with water perfusion and without altering core temperature. This study seems to be crucial to understanding the importance of mattress firmness; in fact, a mattress which is too firm does not let shoulders sink into the mattress, consequently leading to a lack of adequate support to neck and shoulders that causes pain and joint stiffness. On the other hand, in mattresses that are too soft, hips and shoulders sink too much into the mattress, leading to column misalignment. The study concluded that a customized mattress (customized inflation) is able to provide column with higher support during sleep on side position $[4,15]$. Krauchi et al. in their study underlined once more the importance of room temperature in influencing sleep, even though it is still 


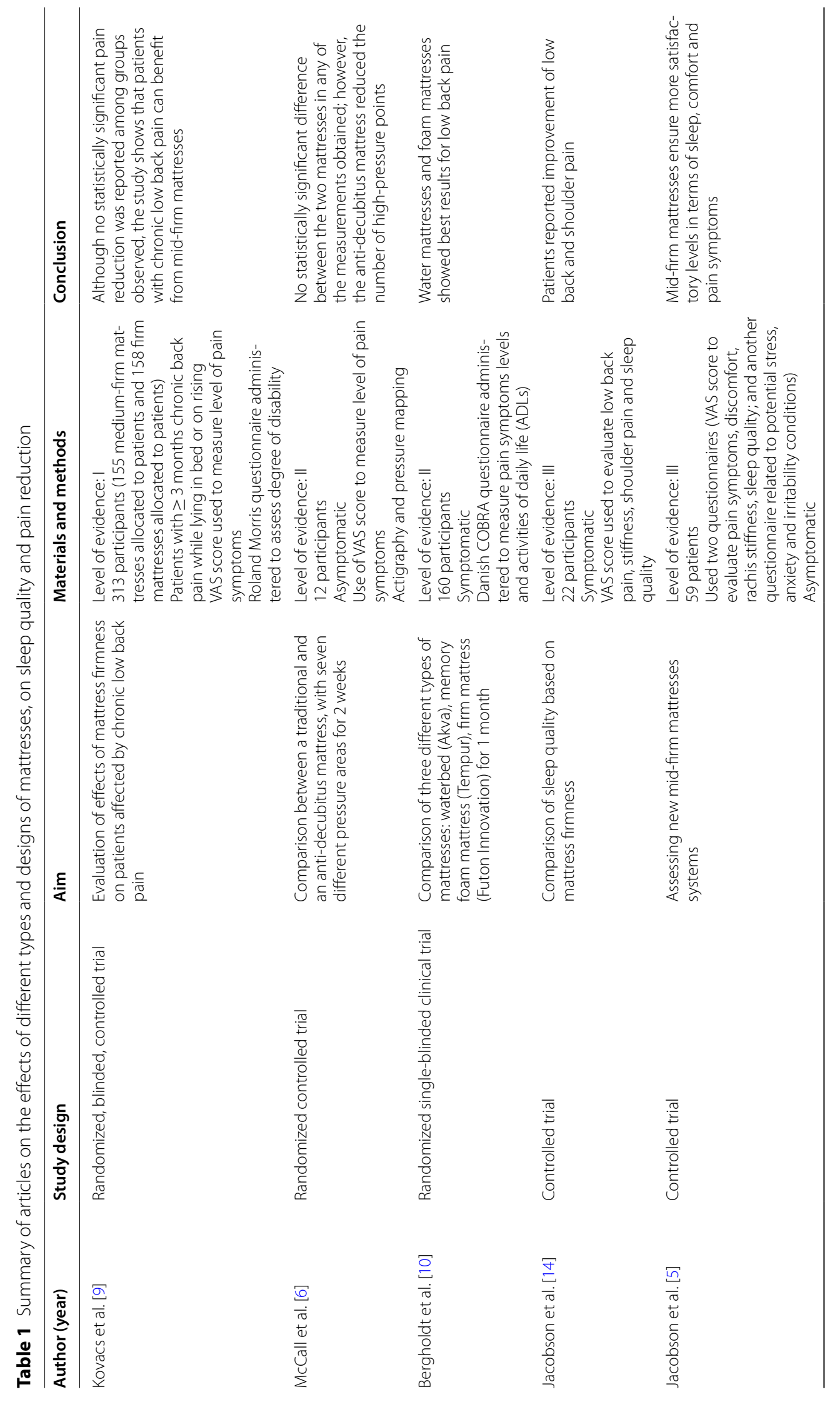




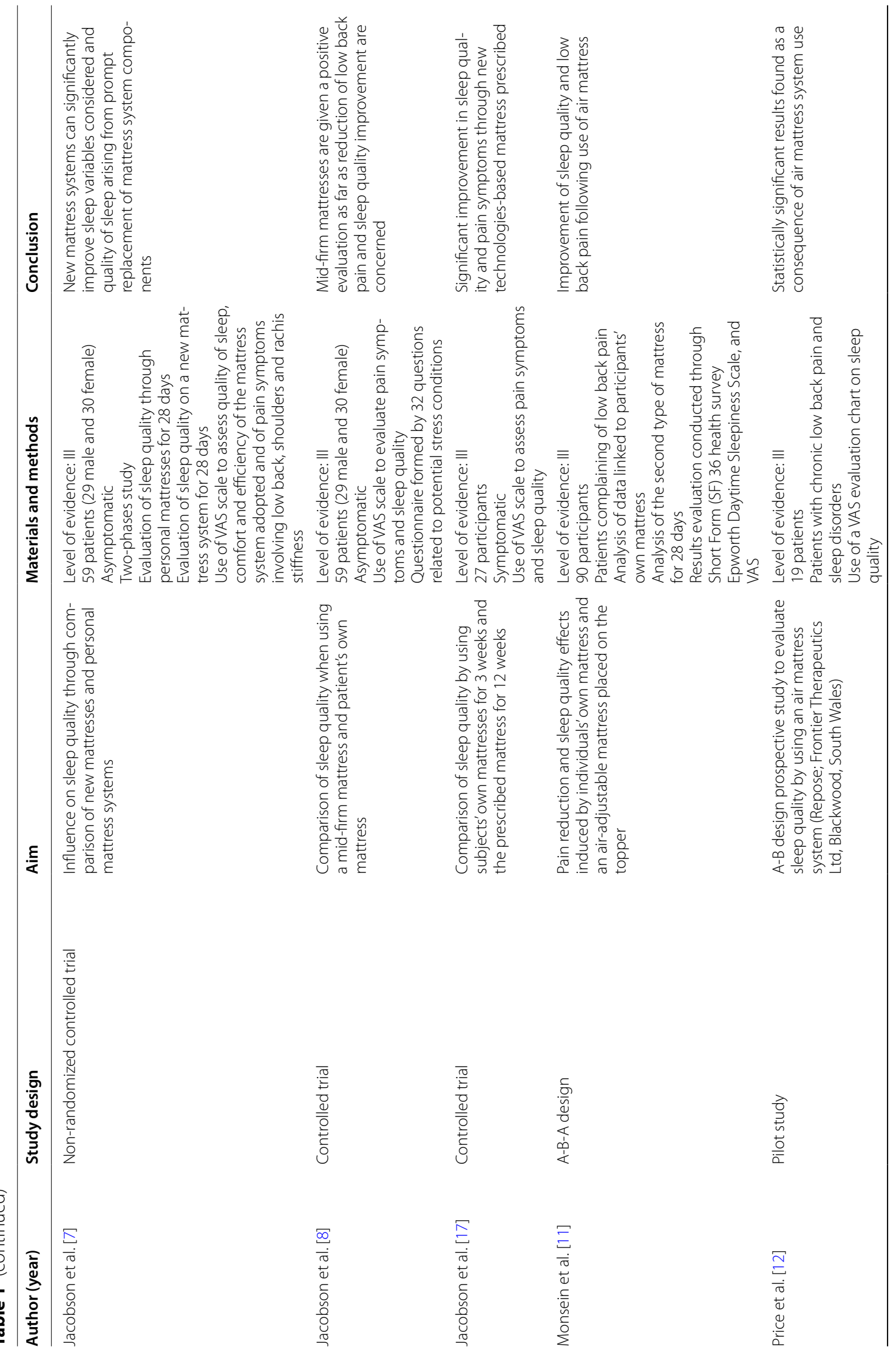



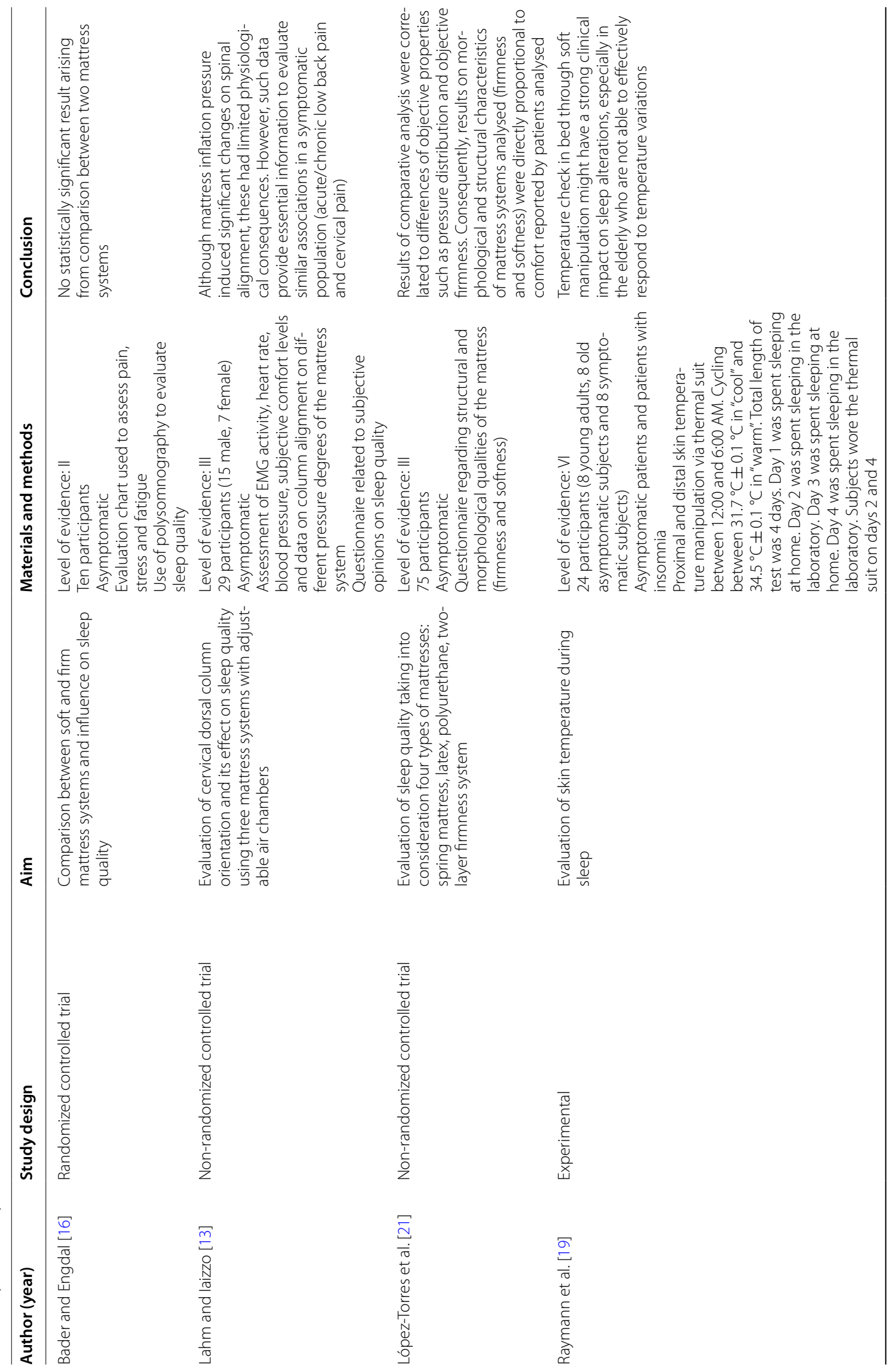


\begin{tabular}{|c|c|c|c|c|}
\hline & 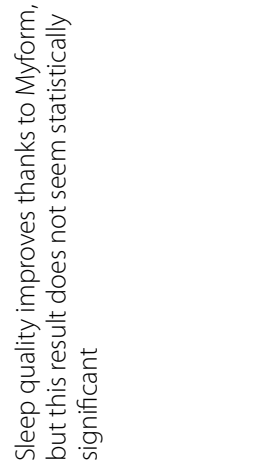 & 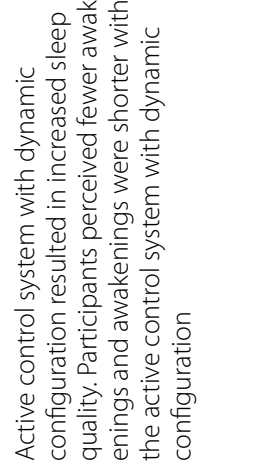 & 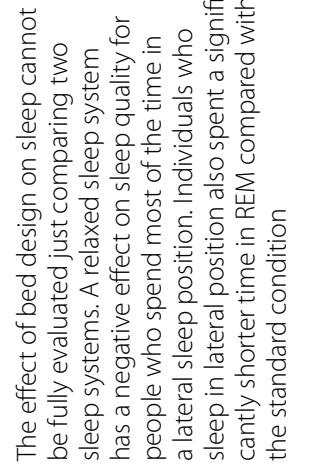 & 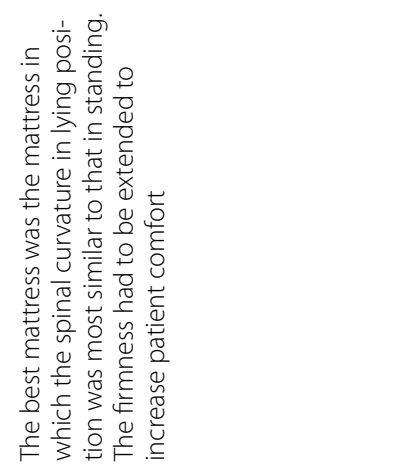 \\
\hline & 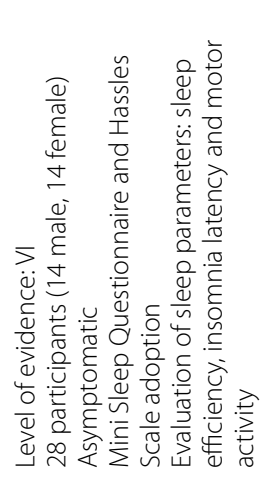 & 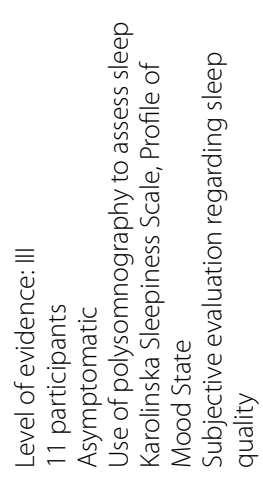 & 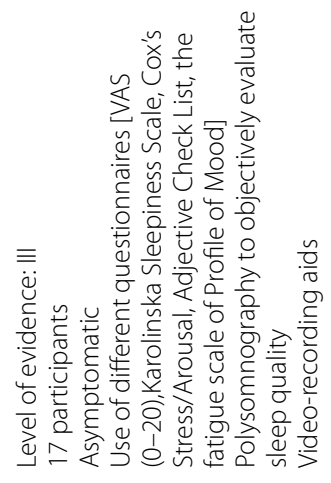 & 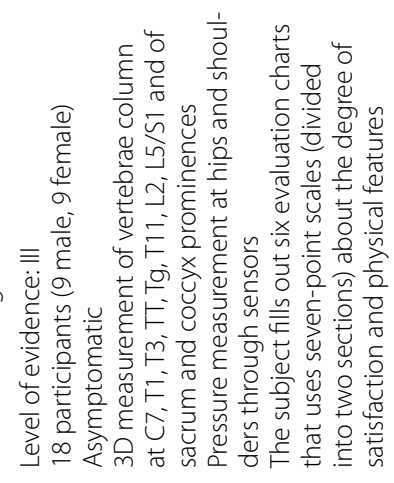 \\
\hline$\frac{\xi}{<}$ & 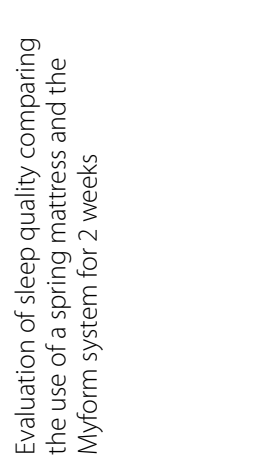 & 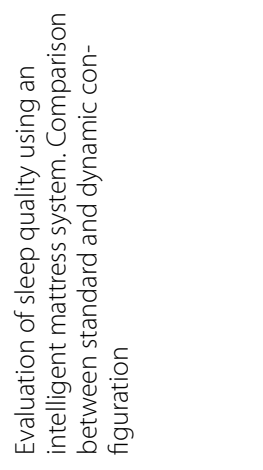 & 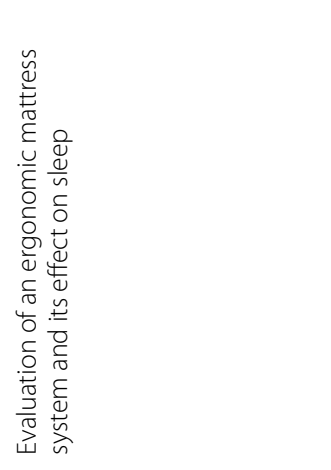 & 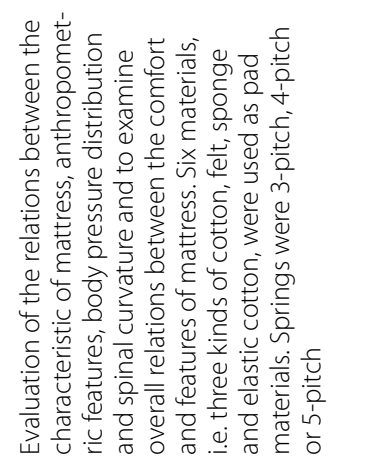 \\
\hline & 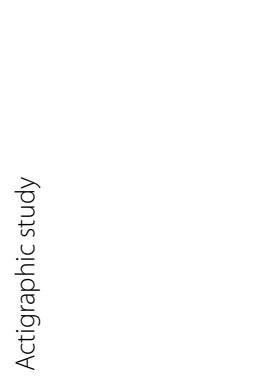 & 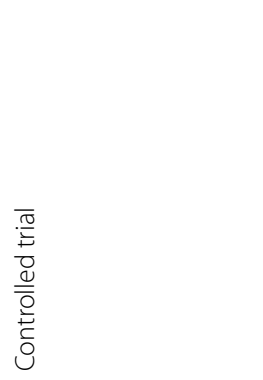 & 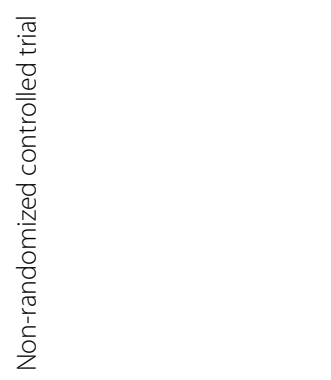 & 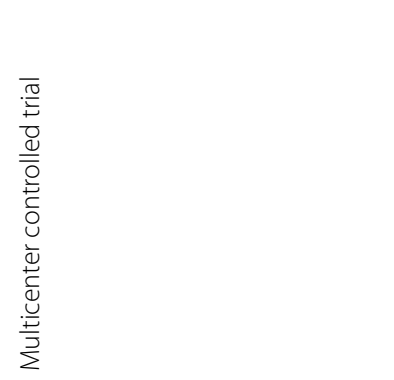 \\
\hline & 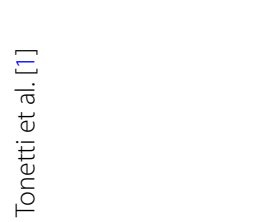 & 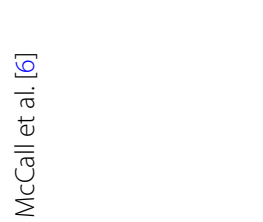 & 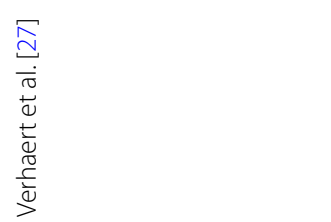 & 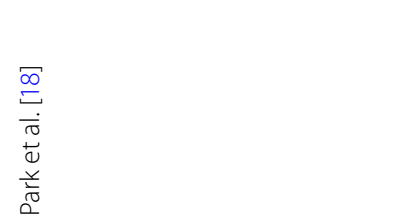 \\
\hline
\end{tabular}




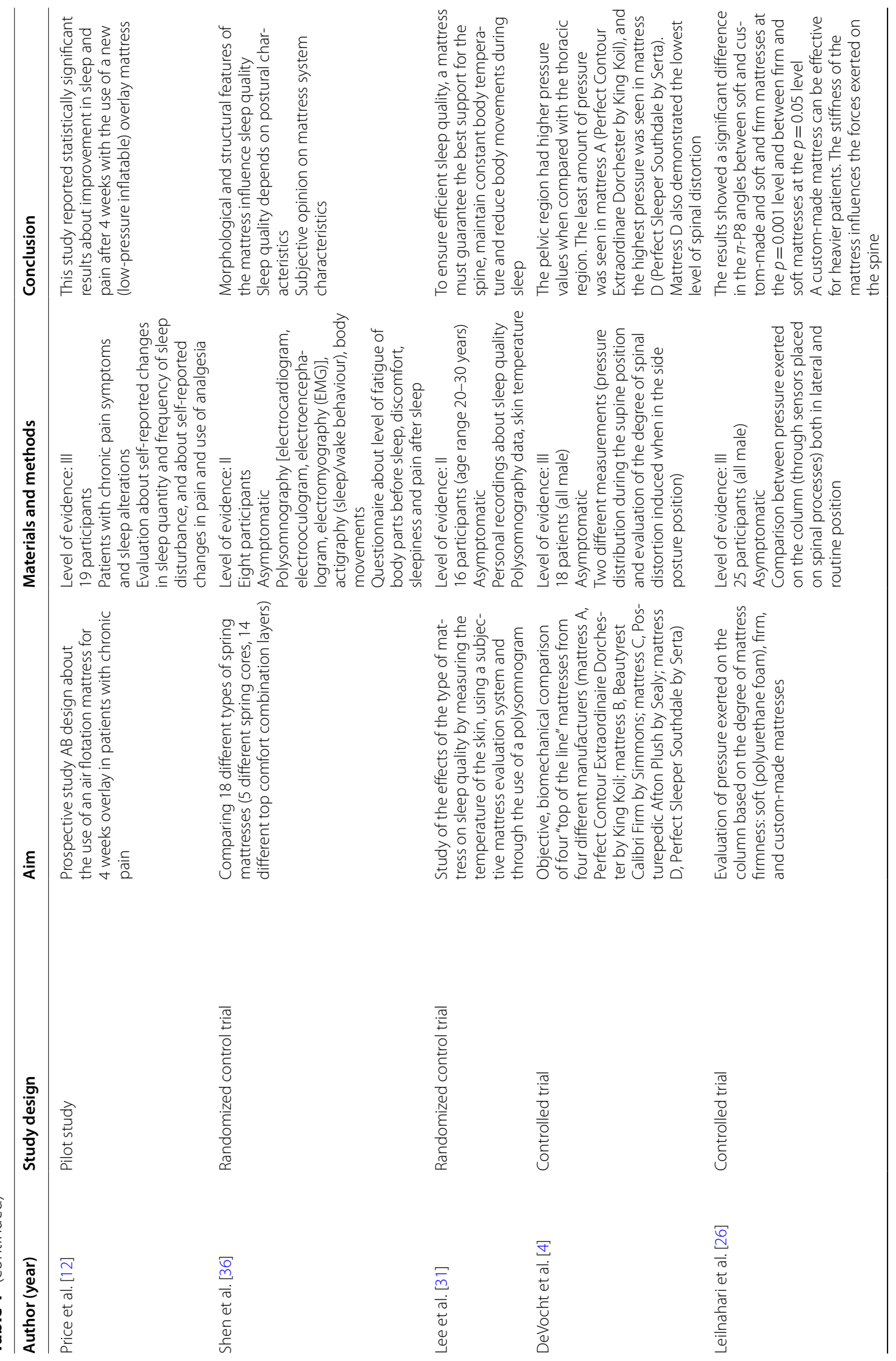




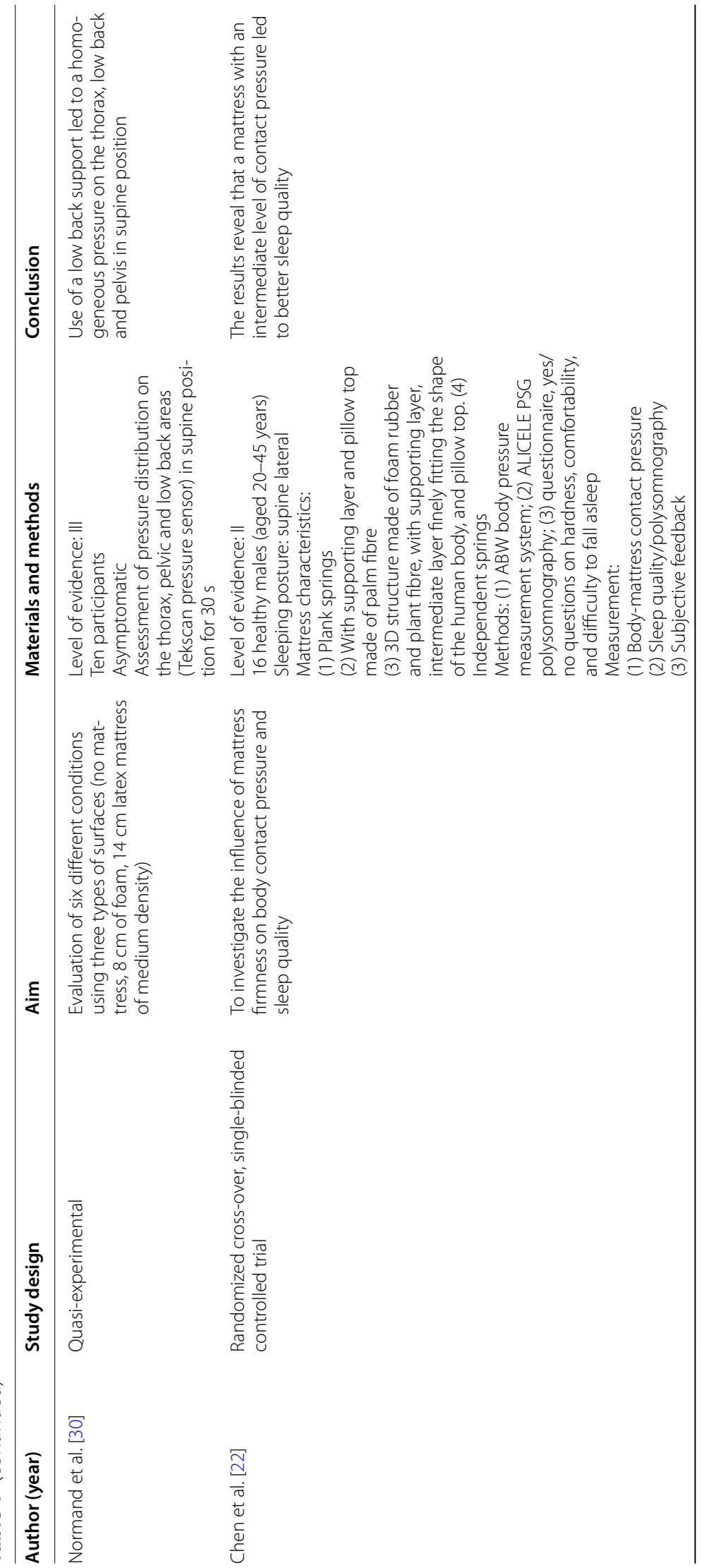




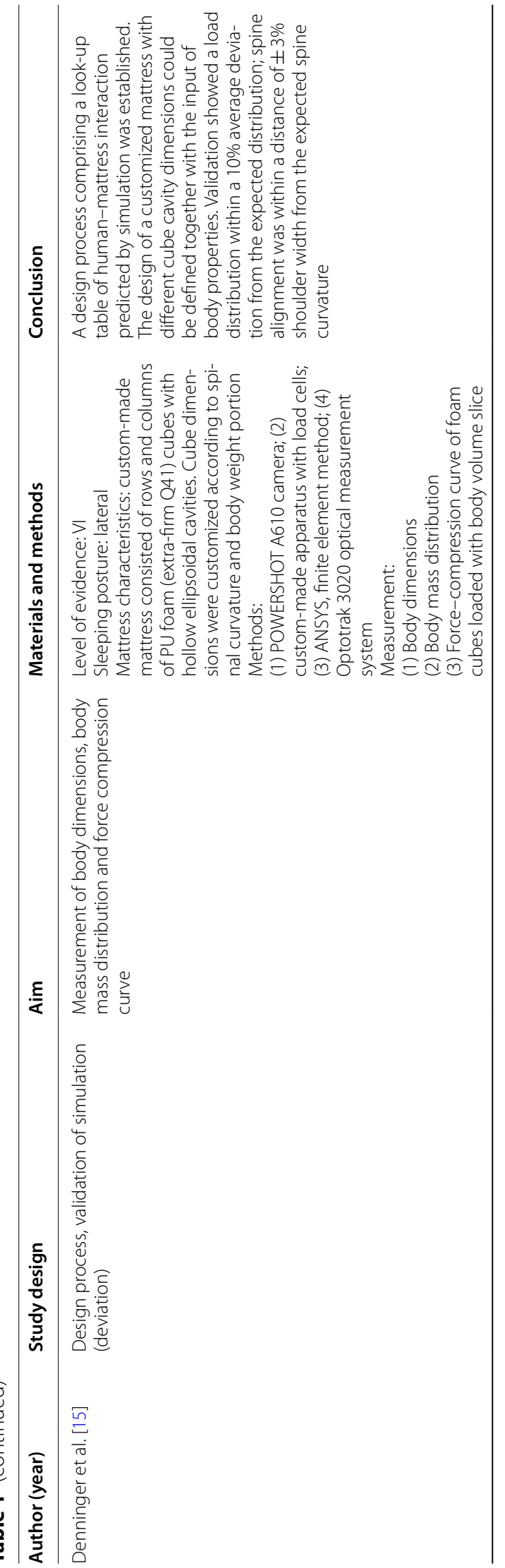




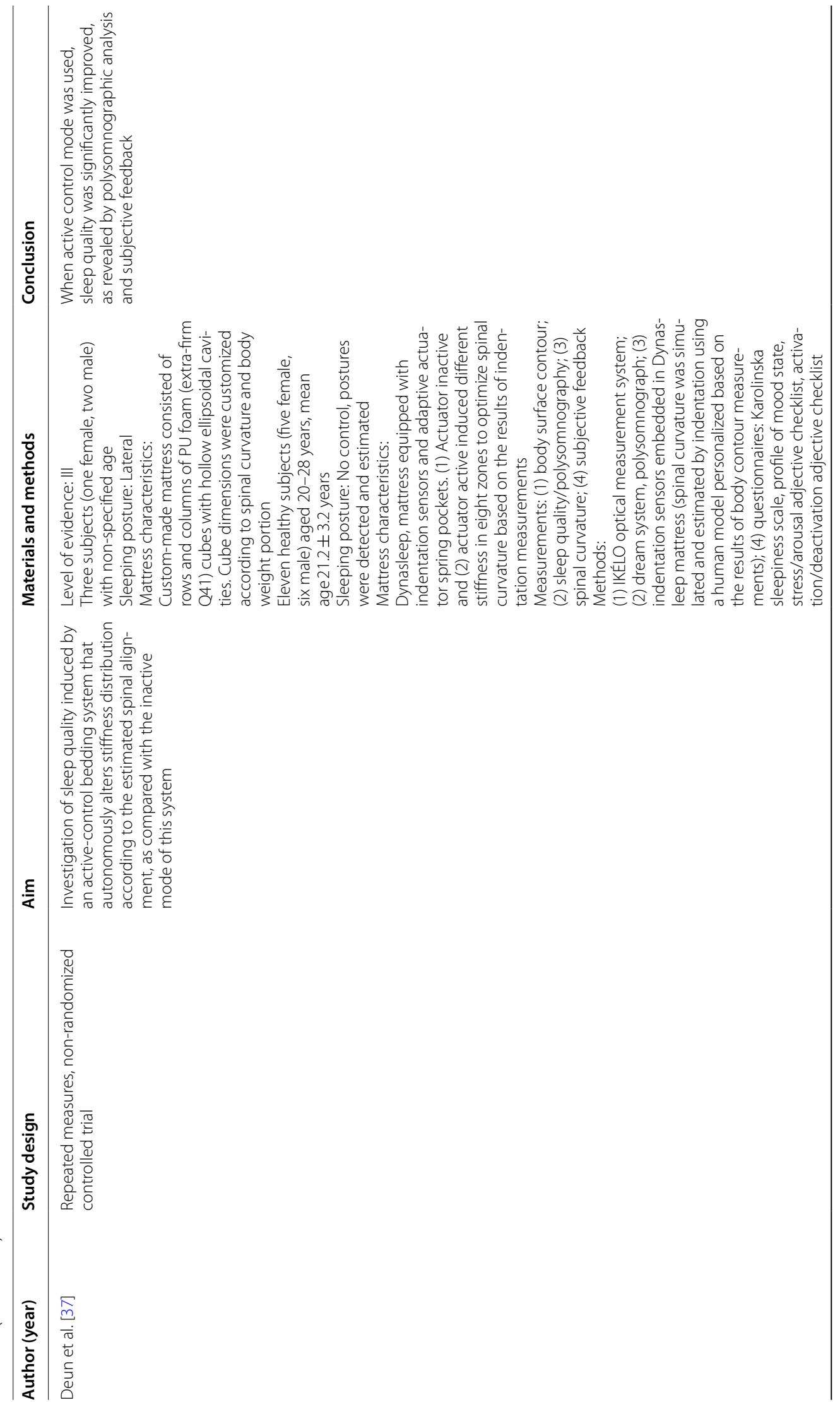




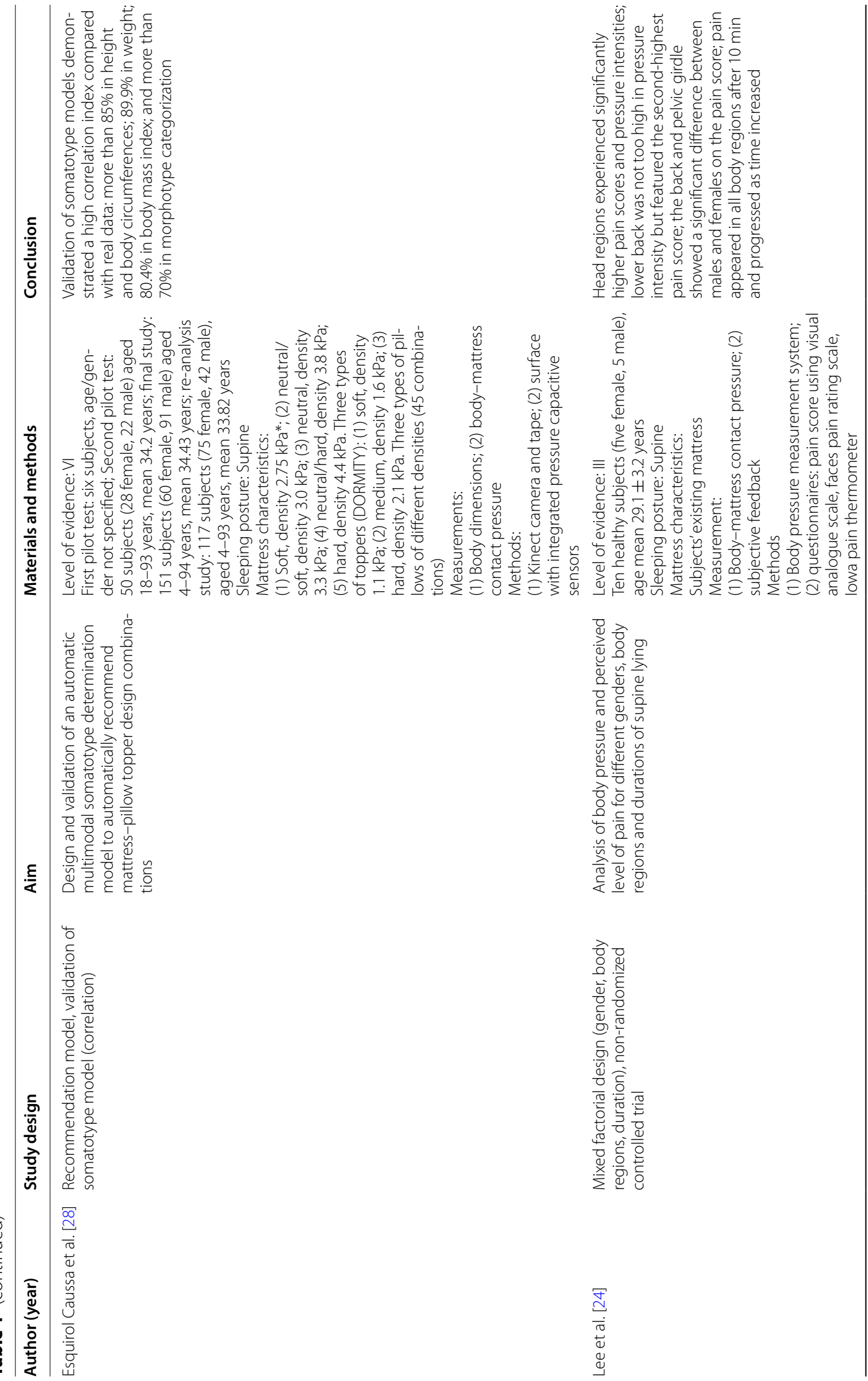




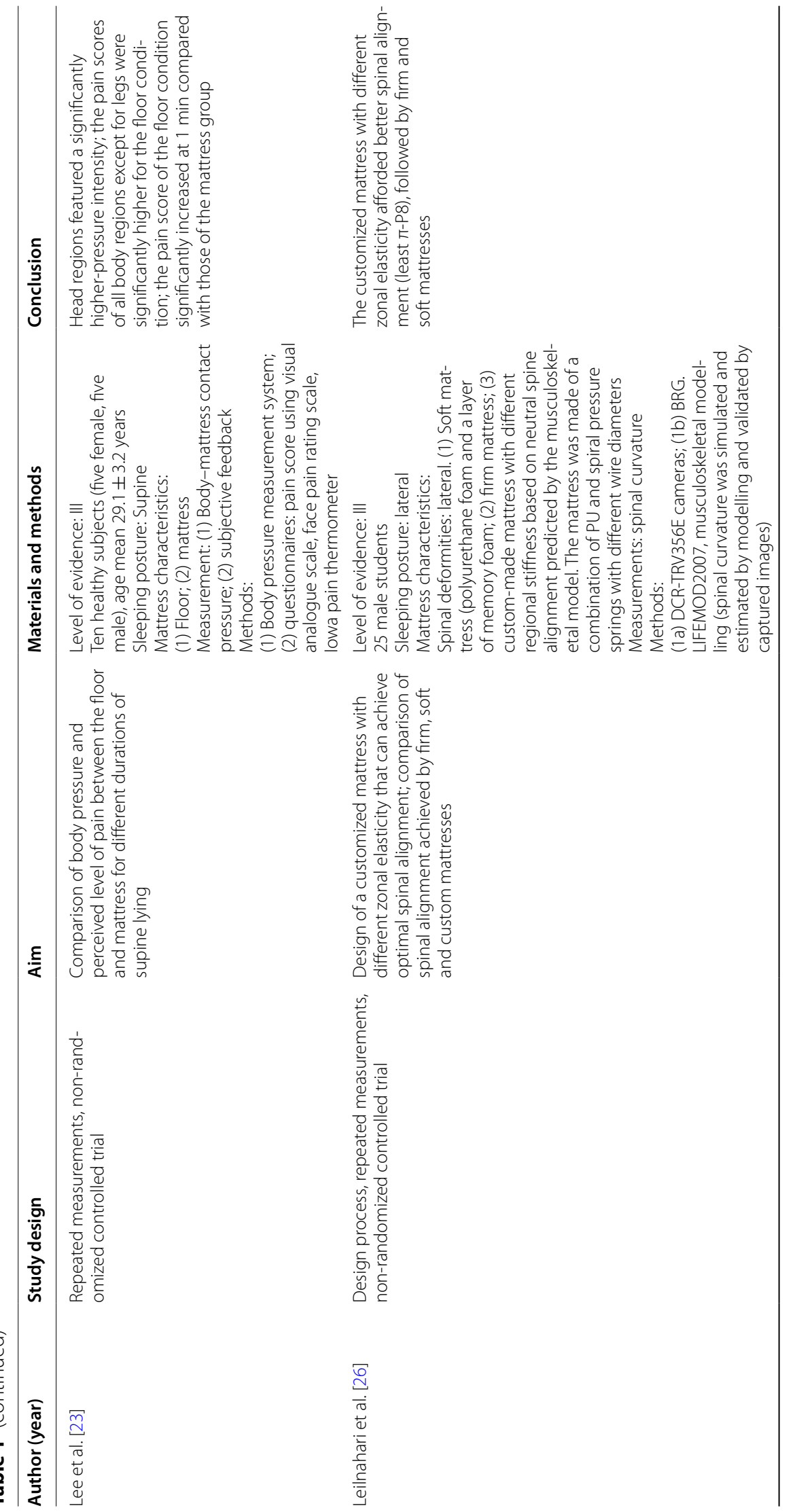




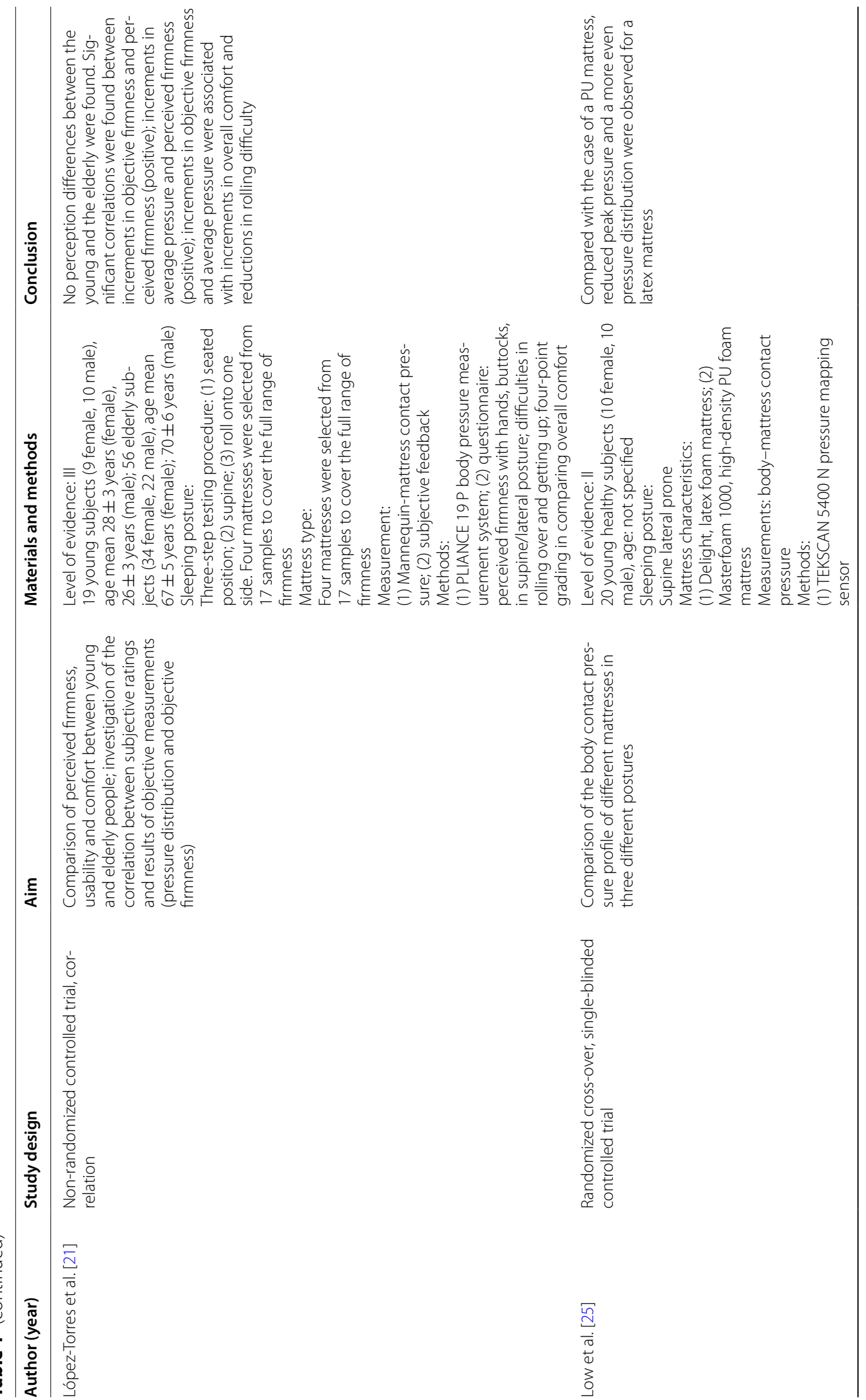




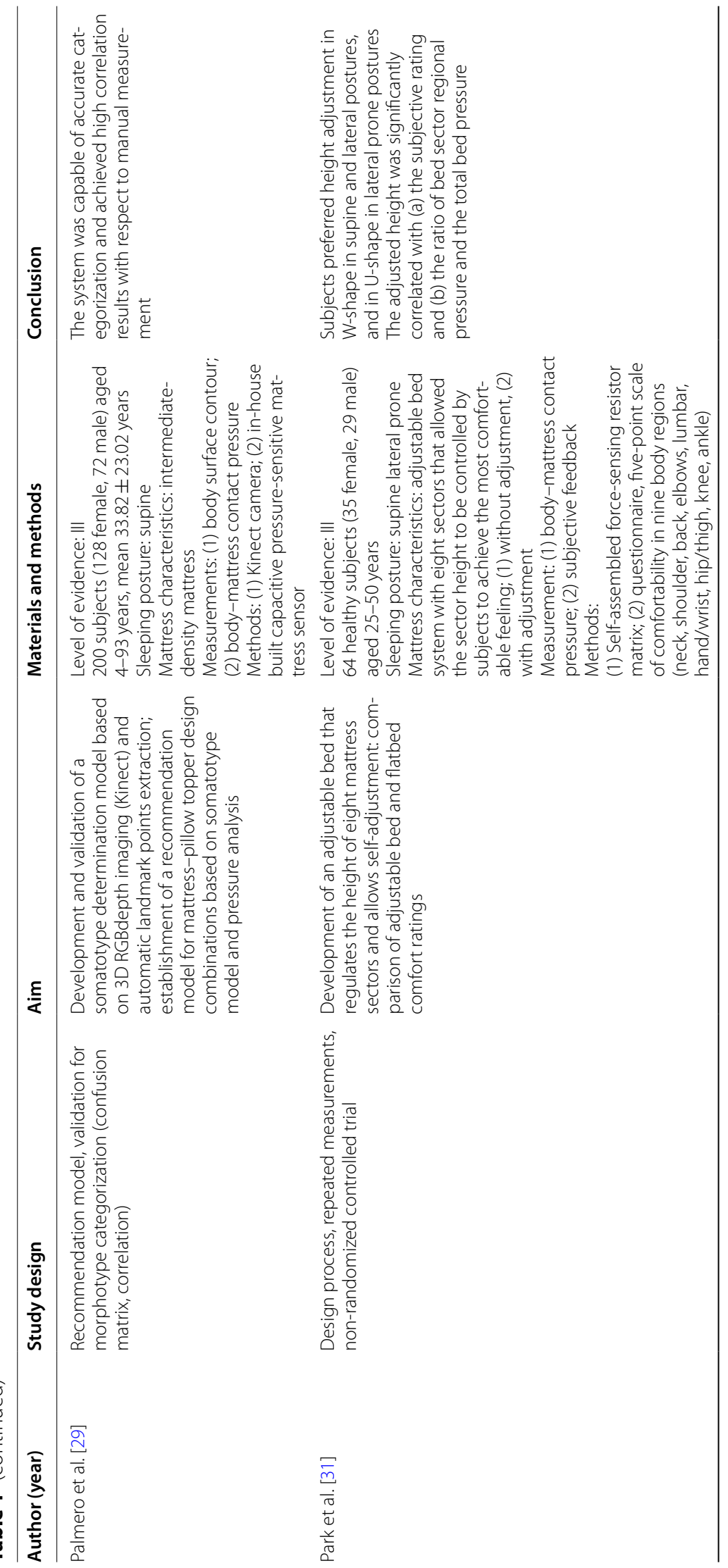




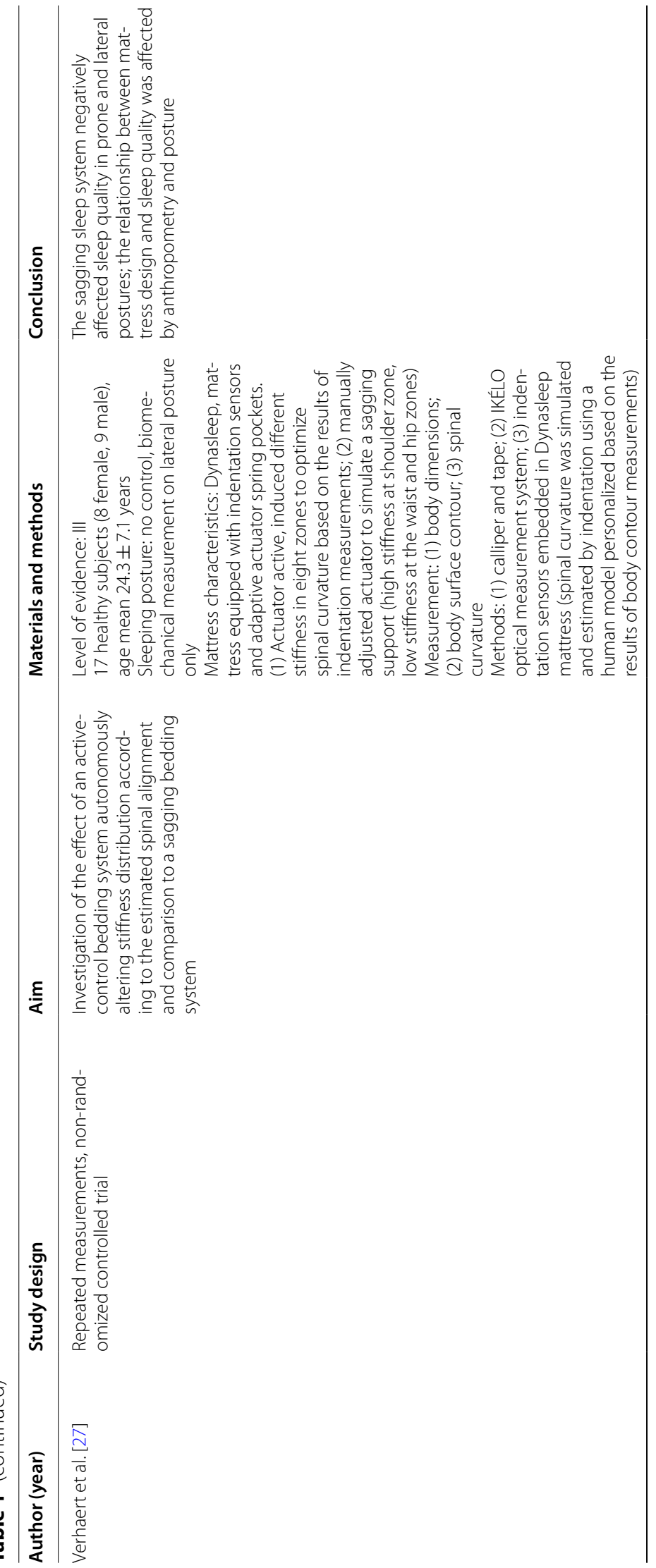




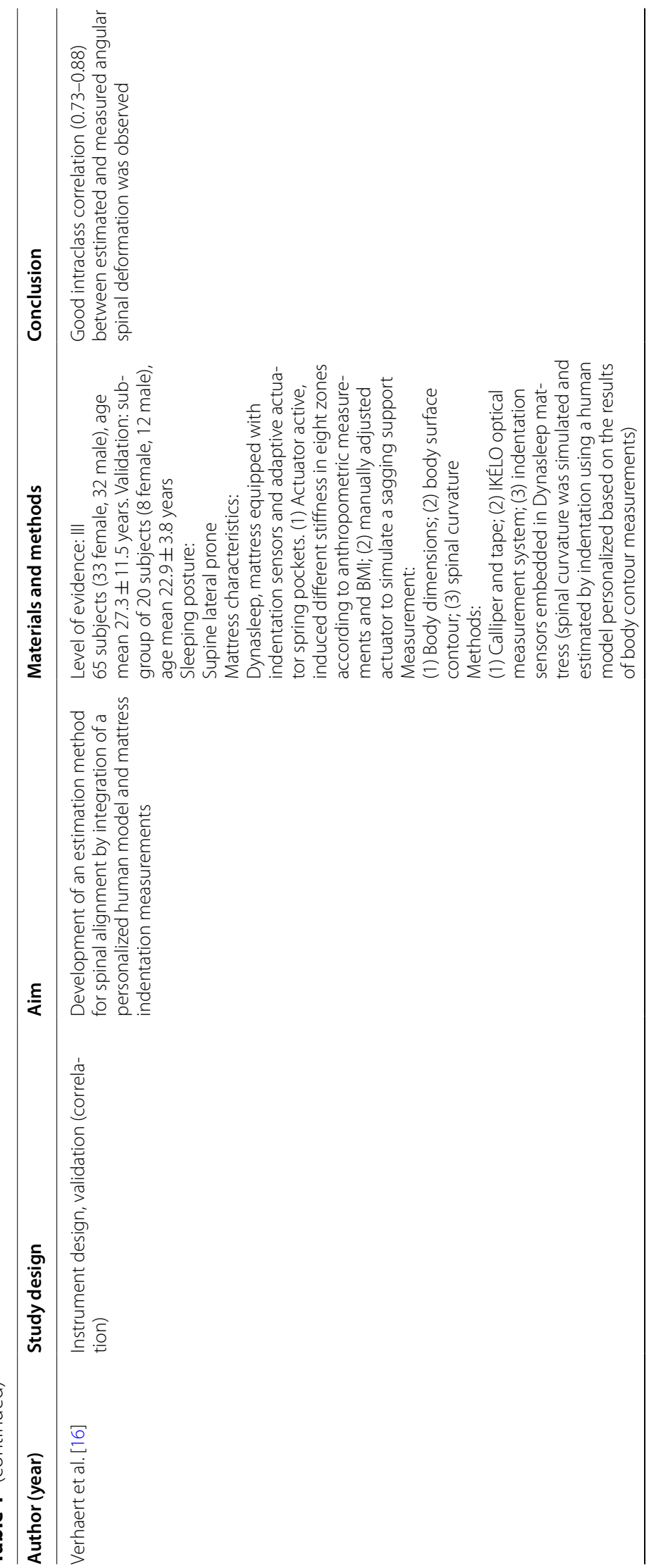




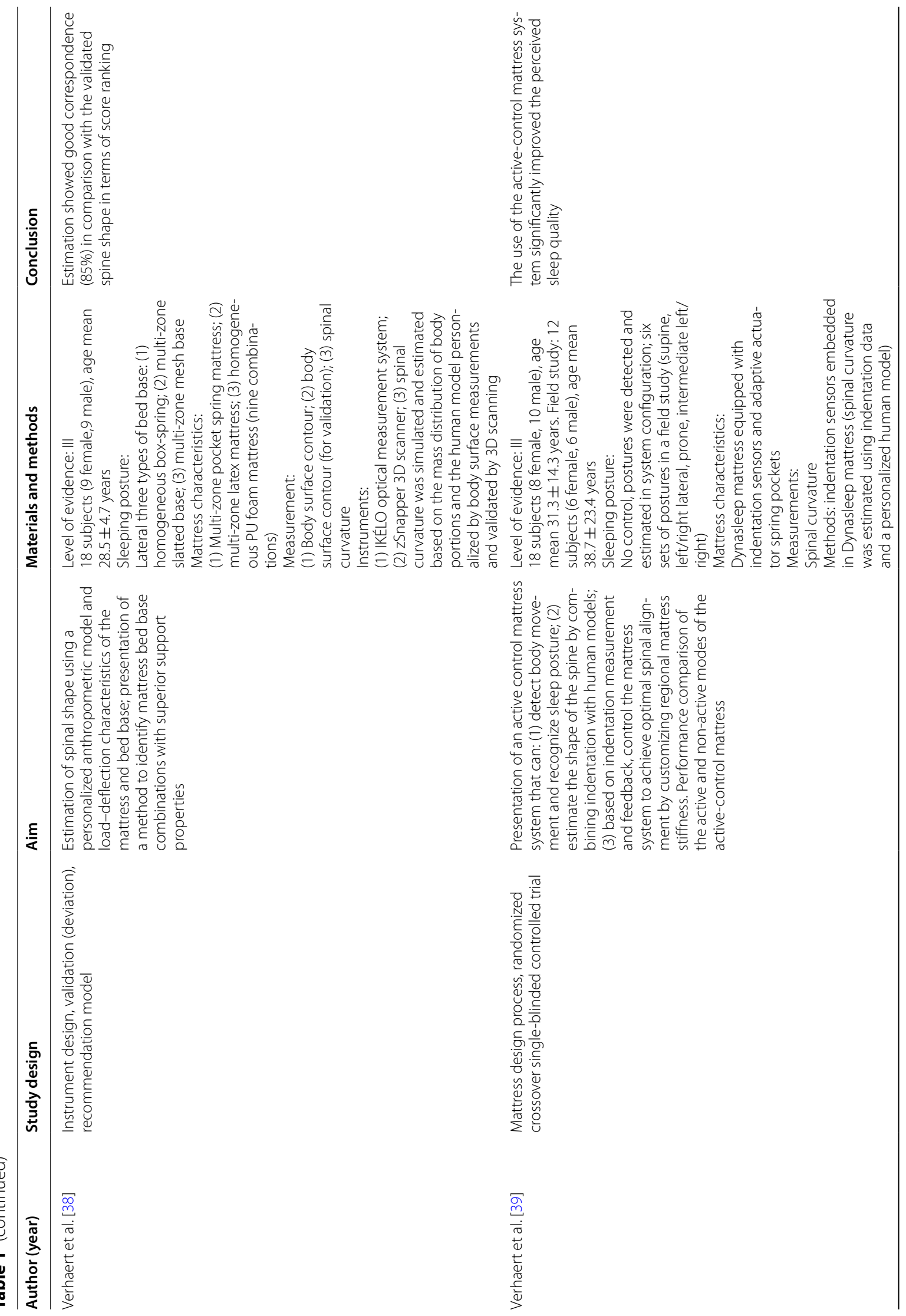




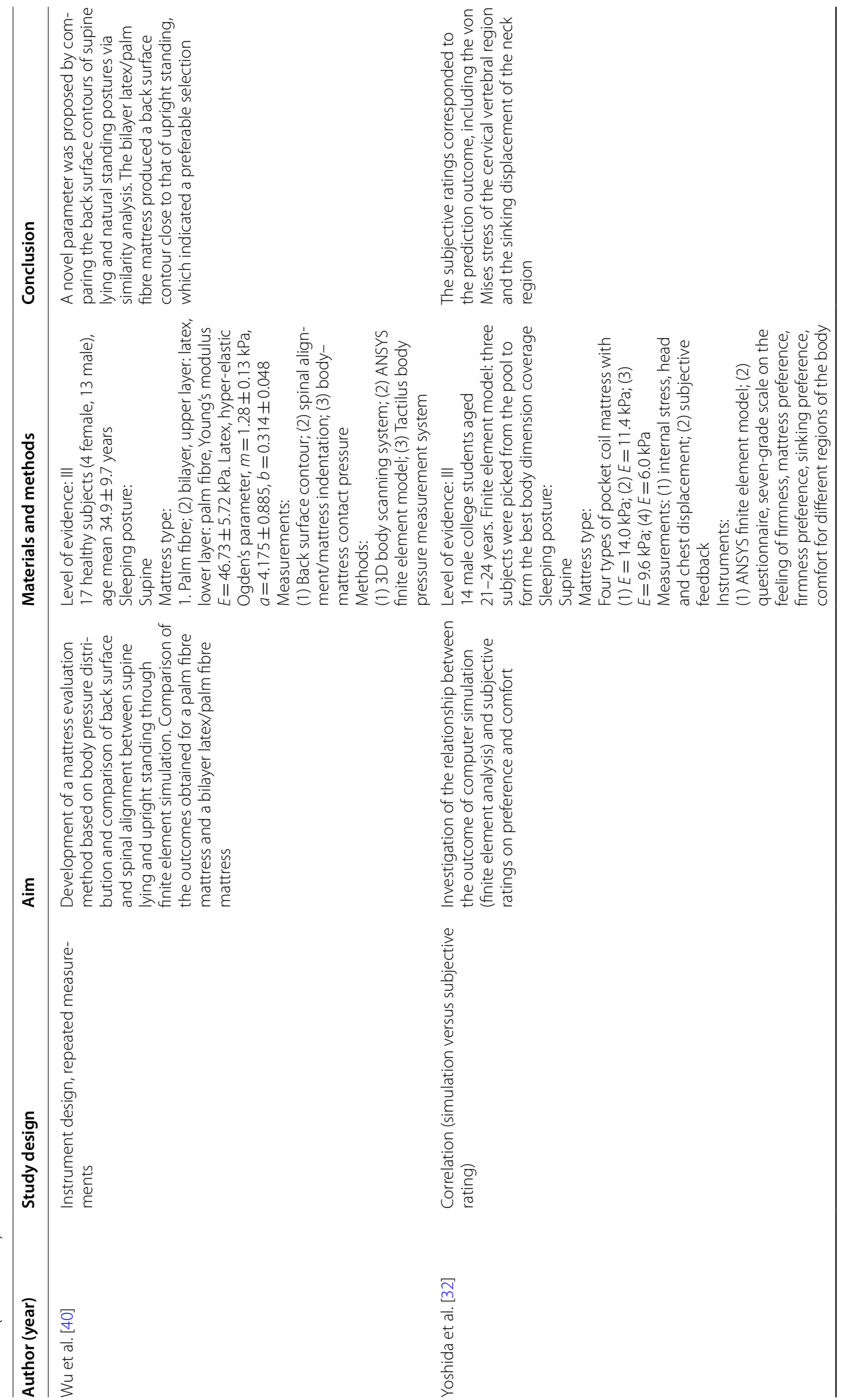




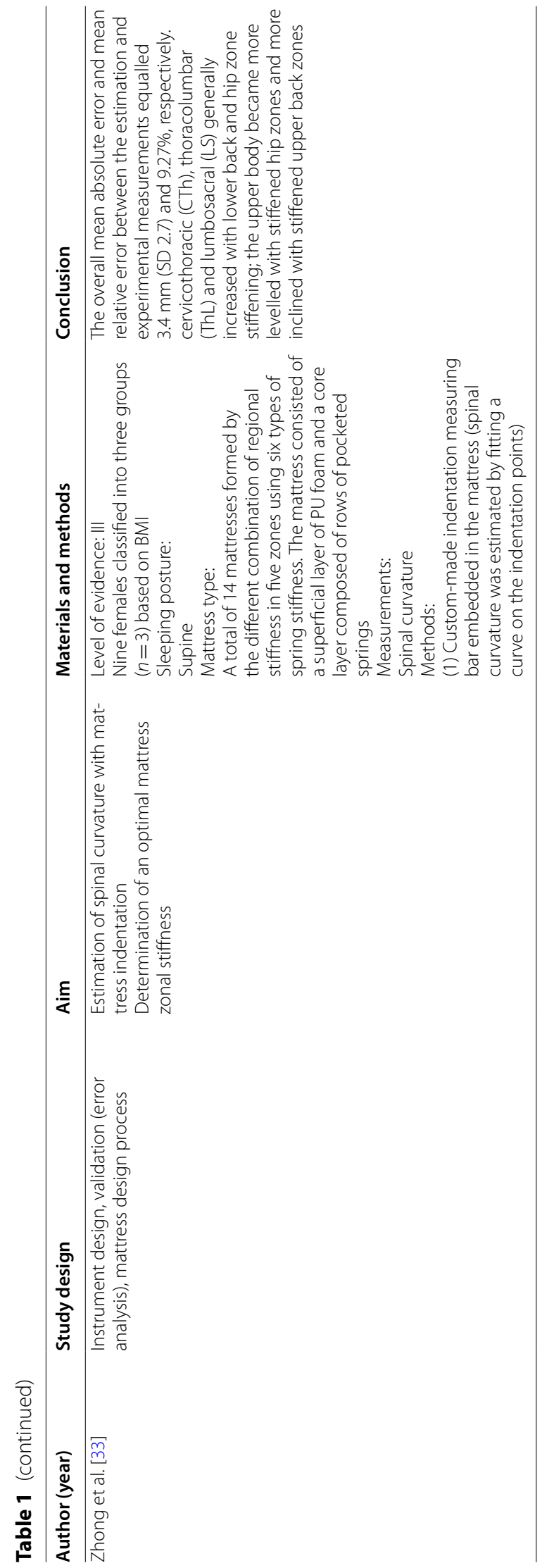


unclear how this occurs. Results showed an improved rachis alignment in beds adapted by an active control that adapted beds, supports and mattresses to the morphology of the examined rachis [16].

\section{Discussion}

In the Jacobson et al. study, 59 healthy subject were enrolled: active individuals, free from known musculoskeletal pathologies, who had been sleeping on commercial spring mattresses for the past 5 years; however, they had occasionally reported physical discomfort during sleep, back stiffness once awake and poor sleep quality, even though no pathological condition associated with sleep had been diagnosed. Volunteers from this study were asked to fill a questionnaire daily for 28 days to investigate back pain, shoulder pain, column stiffness, quality, comfort and sleep efficiency $[5,7,8]$. The same questionnaires were administered to the same subjects during the following 28 days after having slept on a medium-firm mattress delivered at their households. The mattress had the following characteristics: mediumfirm surface based on these components: foam-encased Bonnell spring unit, densified fibre pad, super-soft foam, damask cover, semi-flex foundation, slick fibred; they had the same size as mattresses previously used by volunteers. Results revealed significant improvement for all parameters previously indicated [14].

At the end of the experimental phase at day 28, a back pain decrease of approximately $48 \%$ and an improvement of sleep quality of $55 \%$ were reported; such improvement correlated with a significant decrease in stress levels.

At months 5-6 from the initial experimental phase, individuals were subjected to an additional assessment to complete the evaluation, reporting whether positive effects obtained had lasted over time. In addition, higher BMI was associated with worse sleep quality in both phases [8].

Kovacs et al. conducted a study with 313 adults with non-specific chronic low back pain upon waking up. Mattress firmness was scored based on the scale developed by the European Committee for standardization and went from 0 (maximum firmness) to 10 (minimum firmness). Firm $(\mathrm{Hs}=2 \cdot 3)$ and medium-firm $(\mathrm{Hs}=5 \cdot 6)$ mattresses were randomly allocated to patients. Patients were analysed at time 0 and at 90 days through a visual analogue scale (VAS) scale upon waking up and after $30 \mathrm{~min}$ and with a Spanish version of the Roland Morris questionnaire to evaluate the degree of disability experienced during daily activities.

In a study by Monsein et al., patients were asked to complete a Short Form 36 Health Survey Questionnaire (SF-36) and VAS scale at three different times: after sleeping in their own bed, after 28 days spent on a spring bed with an air topper and then after a period of sleep in their own beds again for 14 nights.

The majority of studies assessed mattress firmness on the basis subjective evaluations, except Kovacs et al., who applied the European Committee Standardization Scale of firmness of mattresses [9]. Subjects from all abovementioned studies used a reproducible grading scale to assess back pain and sleep quality.

However, contrasting results can be found in the literature.

Several authors evaluating the relationship between mattress design and sleep quality studied materials and structural properties of spring mattresses [17]. No significant correlation was found between mattress firmness and sleep quality. However, authors described that a deeper and more effective sleep was observed with softer mattresses, having specificities related to the characteristics of each subject.

Tonetti et al. compared a latex mattress and a traditional spring mattress in 16 healthy volunteers, evaluating pre- and post-results of both through an actigraphy and a Mini Sleep Questionnaire (MSQ).

An objective improvement in sleep efficiency, sleep onset latency and motor activity during sleep was reported for both mattresses; however, no improvement in sleep quality subjective perception was highlighted for the latex mattress.

Likewise, Park et al. evaluated the correlation between anthropometric characteristics, body weight pressure distribution, spinal curve and characteristics of mattresses. In this study, six types of material were used for the mattresses: three different types of cotton, felt, sponge and elastic cotton. The column curve was measured both supinely and standing up through tridimensional measurements. Pressure measurements were taken by using sensors distributed from shoulders to hips. Patients were asked to classify each surface with a specific seven-point score for a subjective evaluation. Based on results from this study, the best mattress was that whose materials and design ensured a rachis curve similar to what usually showed while standing up [18].

Raymann group evaluated that an increase of just $0.4^{\circ}$ led to a decrease in night awakenings and an improved sleep quality, also highlighted by an increase in slow wave activity recorded by electroencephalography [19]. This study was a pioneer for other studies to develop possible thermoregulation systems to be inserted inside a mattress.

Another parameter studied in the literature is the body-mattress contact pressure, commonly associated with pain level and discomfort $[7,20]$. The system to measure body pressure was characterized by sheet sensors, so thin and flexible that they just minimally 
interfered with mattresses [21-24]. However, sensors can disperse concentrated pressure anyway and then underestimate the pressure peak. Similarly to the contact pressure principle, some studies analysed the loading effects of support in some areas using a load cell matrix. Peak pressure, mean pressure and contact area were often measured in different body sites to judge whether pressure was reduced. These tools were used by Fan-Zhe Low to demonstrate that, compared with polyurethane (PU) mattresses, latex mattresses better distribute body pressure points when lying down, reducing peaks involving thorax and sacral areas [25].

Spine alignment was the second most studied parameter, alongside body-mattress contact pressure. Physiological sagittal and coronal planes, in fact, reduce musculoskeletal pain. In the past, several studies carried out measurements of body alignment focusing on only two dimensions by using a video camera. Recently, some studies attempted to perform tridimensional (3D) measurements using an eye-tracking system through a webcam equipped with a depth sensor/infrared projector (Kinect, Microsoft, Redmond, WA, USA) to achieve a recording of images on both sagittal and coronal planes [15, 25-29], [30-33].

To evaluate sleep characteristics through objective data, some researchers identified a number of pressure points on the column that might be considered additional decisive factors to prescribe a mattress. Furthermore, patients were subjected to polysomnography and to a constant measurement of body temperature during sleep. Comfort was evaluated based on the body pressure on the mattress and on the column curvature both standing up and lying down. In this case, mattresses were evaluated as comfortable when during sleep they exhibited a curve with angles similar to the column curvature while standing up. As a result, the most comfortable mattresses that guaranteed also a better sleep quality were those that maintained a higher body temperature during sleep, at the same time supporting the column curve and minimizing unnecessary body movements $[15,34]$. Based on findings achieved by studies previously illustrated, other research groups introduced a concept of "customized mattress" according to the posture of each subject.

In this study, soft, firm and customized mattresses were tested by applying sensors on participants' spinous processes to record rachis alignment through an eye-tracking system during lateral position at rest for each of the three types of mattresses.

The objective was to evaluate how different types of mattresses modify rachis morphology in maximum pressure points in both supine and side positions. Mattresses used were: Perfect Contour Extraordinaire Dorchester By King Koil, Beautyrest Calibri Firm by Simmons;
Posturepedic Afton Plush by Sealy; Perfect Sleeper Southdale by Serta. As expected, the pelvic area presented with higher values compared with the thorax. The best distribution of body contact pressure was attained by the Perfect Contour Extraordinaire Dorchester by King Koil, while The Perfect Sleeper Southdale by Serta showed a lower rachis curve alteration but higher contact pressure involving points of maximum pressure in both supine and prone positions [4].

Krauchi et al. used inclusion and exclusion criteria in their study. Twenty-eight male subjects aged between 25 and 30 years (BMI $19-25 \mathrm{~kg} / \mathrm{m}^{2}$ ) were included in their study and evaluated through a sleep assessment chart. Exclusion criteria were: unsettled severe diseases, history of alcohol or illegal substances abuse, neurological disorders, cranial trauma and mental disorders according to the Diagnostic and Statistical Manual of Mental Disorders (DSM-V, American Psychiatric Association, 2013); other exclusion criteria were: Mini Mental Test Score $<26$, administration of drugs affecting the central nervous system (CNS), excessive consumption of caffeine and/or tobacco (more than two cups and five cigarettes per day, respectively). All volunteers without anamnesis for sleep disturbance and normal sleep (MorningnessEveningness Questionnaire: "none of the two types" [35]) were subjected to an in-hospital polysomnography (PSG) and a long-term monitoring of sleep-wake rhythm evaluated to exclude both sleep disorders (i.e. insomnia, sleep disorders caused by motor activity and or breathing disorders) and assess routine sleep duration and period of sleep. PSG was carried out using a portable monitoring device (Embletta MPR PG proxy ST+, Natus Medical Inc., Pleasanton, California, United States), four-channel electroencephalography (EEG) (C3, C4, O1, O2), two electrooculography chin and tibial electromyography, air flow nasal cannula, abdominal and thoracic breathing effort, oxygen saturation and heart rate, body position and snoring.

Routine sleep time, sleep onset latency, and sleep quality and efficiency were evaluated by wrist actigraphy for 2 weeks (software ActiGraph wGT3X and ActiLife + Sleep software, ActiGraph, Pensacola, FL, USA) together with sleep records. A high-heat-capacity mattress was tested in this study, in which foam layers at the bottom are covered by high-thermal-conductivity polyurethane layers (Technogel, Italia S.R.L., Vicenza, Italy), while the low-heat-capacity mattress is formed $100 \%$ by foam. For both mattresses, measures were $90 \times 200 \times 25 \mathrm{~cm}$. The different thermal behaviour in HHCM and LHCM is correlated to the different density of the $2 \mathrm{~cm}$ covering the most superficial layer (HHCM, $1006 \mathrm{~kg} / \mathrm{m}^{3}$; LHCM, $80 \mathrm{~kg} / \mathrm{m}^{3}$ ) and then related to the different specific thermal capacity in the 
observed temperature interval of $23-35{ }^{\circ} \mathrm{C}$ (HHCM, about $47 \mathrm{~kJ} /{ }^{\circ} \mathrm{C}$; $\mathrm{LHCM}$, about $5,4 \mathrm{~kJ} /{ }^{\circ} \mathrm{C}$ for the $2 \mathrm{~cm}$ of the external surface). To exclude the potential effect of different overlays, the same type of overlay was used during the whole study (bi-elastic non-quilted fabric weighing $600 \mathrm{~g} / \mathrm{m}^{2}$ ). This study also analysed temperature. To measure room, skin and mattress temperature, wireless temperature sensors were used (DS 1922L, Thermochron iButtons; Maxim, Dallas, USA; resolution $0.0625^{\circ} \mathrm{C}$; sampling frequency one value per minute).

Video polysomnography was performed by Comet XL Lab-based PSG (Grass Telefactor, Astro-Med Inc., West Warwick, RI 02893 USA) using four-channel EEG (C3, $\mathrm{C} 4, \mathrm{O} 1, \mathrm{O} 2$ ), two electrooculogram channels (right and left outer canthus), chin electromyogram, heart rate, oxygen saturation and body position.

Temperature in the room was kept around $23{ }^{\circ} \mathrm{C}$ with $\mathrm{a} \pm 0.5{ }^{\circ} \mathrm{C}$ interval and relative humidity between $45 \%$ and 55\%. PSG variables included: time spent in phases N1, N2, N3 (slow wave sleep, SWS), rapid eye movement (REM) sleep, total sleeping time, sleep efficiency and time of wake-up after sleep onset.

The most important result achieved by this study is that comparison between individuals sleeping on lowthermal-capacity mattresses (LHCM) and high-thermalcapacity mattresses (HHCM) demonstrated a significant reduction in core body temperature (CBT), proximal skin temperature on the back (PROBA) and mattress surface temperature, and a significant increase in N3 sleep phase. Regression analysis selectively revealed a significant association between increased CBT-PROBA (and reduced PROBA) with emphasized N3 sleep phase. The present study conducted in a controlled laboratory demonstrated that sleep characteristics can be influenced by thermal properties of the mattress. Comparison between a traditional mattress LHCM and HHCM mattress showed an increase in temperature of proximal skin on the back and of the core body temperature, and an increase in slow sleep waves and sleep continuity [13].

\section{Conclusions}

Based on data reported by literature, it can be claimed that medium-firm mattresses offer more advantages to subjects with non-specific low back pain. Studies have demonstrated, indeed, that these mattresses improve sleep quality and reduce risk of developing low back pain. Beds with active control improve column alignment and sleep quality. Temperature manipulation using a HHCM mattress caused a reduction in temperature increase of proximal skin on the back and of core body temperature, and an increase in slow sleep waves and sleep continuity; slightly manipulating the skin temperature, the wake-up can be delayed and deep sleep can be favoured.

\section{Limitations}

The articles analysed reported differed with regard to their observations, and standardization of volunteers was low in terms of physical and health characteristics. Type of low back pain was poorly determined.

\section{Acknowledgements}

Authors acknowledge the Italian Society of Orthopaedics and Traumatology (SIOT), Prof. Francesco Falez, Prof. Paolo Tranquilli Leali and the whole Executive Board 2018-2020 for having inspired and supervised the current study.

\section{Authors' contributions}

G.C., G.R.T. and L.P. made substantial contributions to design, analysis and interpretation of data, and contributed to the writing and revising of the manuscript. G.T., E.J. and G.M. contributed to the acquisition and interpretation of data, and collaborated with the writing and revising of the manuscript. All authors read and approved the final manuscript.

\section{Funding}

The research presented has not received any grant funding.

\section{Availability of data and materials}

Studies carried out between 2000 and 2019 have been included in the current review. Mattresses analysed in the studies examined by our research group have been classified according to the European Committee for Standardization (2000) as soft, medium-firm, extra-firm or customized to avoid supine decubitus. All authors read and approved the final manuscript.

\section{Declarations}

Ethics approval and consent to participate

No approval is required for the study because the data are published.

\section{Consent for publication}

All authors consent to the publication of this manuscript.

\section{Competing interests}

The authors report no financial conflicts of interest.

\section{Author details}

${ }^{1}$ Orthopaedic and Traumatology Department, Università degli Studi di Sassari, 07100 Sassari, Italy. ${ }^{2}$ Orthopaedic and Traumatologic Clinic, University of Perugia, Santa Maria della Misericordia Hospital, 06129 Perugia, Italy. ${ }^{3}$ Department of Medical and Surgical Specialties and Dentistry, University of Campania "Luigi Vanvitelli", 80138 Naples, Italy. ${ }^{4}$ Department of Orthopaedics and Traumatology, Fondazione Policlinico IRCCS San Matteo, University of Pavia, 27100 Pavia, Italy. ${ }^{5}$ Orthopaedic, Trauma and Spine Unit, Department of Basic Medical Sciences, Neuroscience and Sense Organs, School of Medicine, AOU Policlinico Consorziale, University of Bari "Aldo Moro", 70100 Bari, Italy. ${ }^{6}$ Orthopaedic and Traumatology Department, Rovereto - Arco Hospital, 38068 Rovereto, Italy.

Received: 7 May 2021 Accepted: 27 November 2021

Published online: 08 December 2021

\section{References}

1. Tonetti LL, Martoni MM, Natale WV (2011) Effects of different mattresses on sleep quality in healthy subjects: an actigraphic study. Biol Rhythm Res 42(2):89-97

2. Swanson LM, Arnedt JT, Rosekind MR, Belenky G, Balkin TJ, Drake C (2011) Sleep disorders and work performance: findings from the 2008 national sleep foundation sleep in America poll. J Sleep Res 20(3):487-494

3. Cimmino MA, Ferrone C, Cutolo M (2011) Epidemiology of chronic musculoskeletal pain. Best Pract Res Clin Rheumatol 25:173-183

4. DeVocht J, Wilder D, Bandstra E, Spratt K (2006) Biomechanical evaluation of four different mattresses. Appl Ergon 37:297-304 
5. Jacobson B, Wallace T, Gemmell H (2006) Subjective rating of perceived back pain, stiffness and sleep quality following introduction of mediumfirm bedding systems. J Chiropr Med 5(4):128-134

6. McCall WV, Boggs N, Letton A (2012) Changes in sleep and wake in response to different sleeping surfaces: a pilot study. Appl Ergon 43(2):386-391

7. Jacobson B, Wallace T, Smith D, Kolb T (2008) Grouped comparisons of sleep quality for new and personal bedding systems. Appl Ergon 39(2):247-254

8. Jacobson B, Boolani A, Smith D (2009) Changes in back pain, sleep quality, and perceived stress after introduction of new bedding systems. J Chiropr Med 8(1):1-8

9. Kovacs FM, Abraira V, Peña A et al (2003) Effect of firmness of mattress on chronic nonspecific low-back pain: randomized, double-blind, controlled, multicentre trial. Lancet 362(9396):1599-1604

10. Bergholdt K, Fabricius R, Bendix T (2008) Better backs by better beds? Spine 33(7):703-708

11. Monsein M, Corbin TP, Culliton PD, Merz D, Schuck EA (2000) Short-term outcomes of chronic back pain patients on an airbed vs innerspring mattresses. MedGenMed 2(3):E36

12. Price P, Rees-Matthews S, Tebble N, Camilleri J (2003) The use of a new overlay mattress in patients with chronic pain: impact on sleep and selfreported pain. Clin Rehabil 17(5):488-492

13. Lahm R, laizzo PA (2002) Physiologic responses during rest on a sleep system at varied degrees of firmness in a normal population. Ergonomics 45(11):798-815

14. Jacobson B, Gemmell H, Hayes B, Altena T (2002) Effectiveness of a selected bedding system on quality of sleep, low back pain, shoulder pain, and spine stiffness. J Manipulative Physiol Ther 25(2):88-92

15. Denninger M, Martel F, Rancourt D (2011) A single step process to design a custom mattress that relieves trunk shear forces. Int J Mech Mater Des 7(1):1-16

16. Verhaert V, Druyts $H$, Van Deun D, De Wilde T, Van Brussel $K$, Haex B, Sloten JV (2012) Modeling human-bed interaction: the predictive value of anthropometric models in choosing the correct bed support. Work 41:2268-2273

17. Jacobson B, Boolani A, Dunklee G, Shepardson A, Acharya H (2010) Effect of prescribed sleep surfaces on back pain and sleep quality in patients diagnosed with low back and shoulder pain. Appl Ergon 42(1):91-97

18. Park SJ, Kim JS, Kim CB (2009) Comfort evaluation and bed adjustment according to sleeping positions. HumanFact Ergon Manufact Ser Ind 19(2):145e157

19. Normand MC, Descarreaux M, Poulin C, Richer N, Mailhot D, Black P, Dugas C (2005) Biomechanical effects of a lumbar support in a mattress. J Can Chiropr Assoc 49(2):96-101

20. Kilinc-Balci F. How consumers perceive comfort in apparel. In: Improving Comfort in Clothing. Amsterdam: Elsevier. 2011; 97-113. https://doi.org/ 10.1533/9780857090645.1.97

21. López-Torres M, Porcar R, Solaz J, Romero T (2008) Objective firmness, average pressure and subjective perception in mattresses for the elderly. Appl Ergon 39(1):123-130

22. Chen Z, Li Y, Liu R, Gao D, Chen Q, Hu Z, Guo J (2014) Effects of interface pressure distribution on human sleep quality. PLoS ONE 9(6):e99969

23. Lee W-D, Lee J-U, Kim M-Y, Lee L-K, Park B-S, Yang S-M, Noh J-W, Shin Y-S, Kim J-H, Kwak T-Y, Lee T-H, Park J, Kim J (2016) Differences in the body pressure-related sensory changes between the floor and mattress in a static supine position for physiotherapy research: a randomized controlled pilot trial. J Phys Ther Sci 28(4):1086-1091

24. Lee W-D, Lee J-U, Park J, Kim J (2015) Analysis of the body pressurerelated sensory changes in the static supine position for healthy science research: a randomized controlled pilot trial. Toxicol Environ Heal Sci 7(3):211-216

25. Low F-Z, Chua MC-H, Lim P-Y, Yeow C-H (2017) Effects of Mattress material on body pressure profiles in different sleeping postures. J Chiropr Med 16(1):1-9

26. Leilnahari K, Fatouraee N, Khodalotfi M, Sadeghein MA, Kashani YA (2011) Spine alignment in men during lateral sleep position: experimental study and modeling. Biomed Eng Online 10(1):103

27. Verhaert V, Haex B, Wilde TD, Berckmans D, Verbraecken J, Valck ED, Sloten JV (2011) Ergonomics in bed design: the effect of spinal alignment on sleep parameters. Ergonomics 54(2):169-178
28. Esquirol Caussa J, Palmero Cantariño C, Bayo Tallón V, Cos Morera MÀ, Escalera S, Sánchez D, Sánchez Padilla M, Serrano Domínguez N, Relats VM (2017) Automatic RBG-depth-pressure anthropometric analysis and individualised sleep solution prescription. J Med Eng Technol 41(6):486-497

29. Palmero C, Esquirol J, Bayo V, Cos MÀ, Ahmadmonfared P, Salabert J, Sánchez D, Escalera S (2017) Automatic sleep system recommendation by multi-modal RBG-depth-pressure anthropometric analysis. Int J Comput Vision 122(2):212-227

30. Manca A, Monticone M, Cugusi L, Doria C, Tranquilli-Leali P, Deriu F (2018) Back surface measurements by rasterstereography for adolescent idiopathic scoliosis: from reproducibility to data reduction analyses. Eur Spine J 27(9):2130-2138

31. Park SJ, Lee H-J, Hong KH, Kim JT (2001) Evaluation of mattress for the Koreans. Proc Hum Factors Ergon Soc Annu Meet. 45(7):727-730

32. Yoshida H, Kamijo M, Shimizu Y (2012) A study to investigate the sleeping comfort of mattress using finite element method. Kansei Eng Int J 11:155-162

33. Zhong S, Shen L, Zhou L, Guan Z (2014) Predict human body indentation lying on a spring mattress using a neural network approach. Proc Inst Mech Eng 228(8):787-799

34. Wang Y, Wong DWC, Zhang M (2016) Computational models of the foot and ankle for pathomechanics and clinical applications: a review. Ann Biomed Eng 44(1):213-221

35. Kräuchi K, Fattori E, Giordano A, Falbo M, ladarola A, Agli F, Tribolo A, Mutani R, Cicolin A (2018) Sleep on a high heat capacity mattress increases conductive body heat loss and slow wave sleep. Physiol Behav 185:23-30

36. Shen $L$, Chen Y, Guo Y et al (2012) Research on the relationship between the structural properties of bedding layer in spring mattress and sleep quality. Work 41 (Suppl. 1):1268-1273

37. Deun DV, Verhaert V, Willemen T, Wuyts J, Verbraecken J, Exadaktylos V, Haex B, Vander SJ (2012) Biomechanics-based active control of bedding support properties and its influence on sleep. Work 41:1274-1280

38. Verhaert V, Druyts H, Van Deun D, Exadaktylos V, Verbraecken J, Vandekerckhove M, Haex B, Vander SJ (2012) Estimating spine shape in lateral sleep positions using silhouette-derived body shape models. Int J Ind Ergon 42(5):489-498

39. Verhaert V, Van Deun D, Verbraecken J, Vandekerckhove M, Exadaktylos V, Haex B, VanderSloten J (2013) Smart control of spinal alignment through active adjustment of mechanical bed properties during sleep. J Ambient Intell Smart Environ. 5:369-380

40. Wu J, Yuan H, Li X (2018) A novel method for comfort assessment in a supine sleep position using three-dimensional scanning technology. Int J Ind Ergon 67:104-113

41. Yamato TP, Maher C, Koes B, Moseley A (2017) The PEDro scale had acceptably high convergent validity, construct validity, and interrater reliability in evaluating methodological quality of pharmaceutical trials. J Clin Epidemiol 86:176-181

42. Hyunja L, Park S (2006) Quantitative effects of mattress types (comfortable vs. uncomfortable) on sleep quality through polysomnography and skin temperature. Int J Ind Ergon 36:943-949

43. Raymann RJEM, Swaab DF, Van Someren EJW (2008) Skin deep: enhanced sleep depth by cutaneous temperature manipulation. Brain J Neurol 131(Pt 2):500-513

\section{Publisher's Note}

Springer Nature remains neutral with regard to jurisdictional claims in published maps and institutional affiliations. 